\title{
Demographic and Clinical Characteristics of Patients with Dementia in Greece
}

\author{
Eleni Jelastopulu, ${ }^{1}$ Evangelia Giourou, ${ }^{1}$ Konstantinos Argyropoulos, ${ }^{1,2}$ Eleftheria Kariori, ${ }^{3}$ \\ Eleftherios Moratis, ${ }^{4}$ Angeliki Mestousi, ${ }^{4}$ and John Kyriopoulos ${ }^{5}$
}

${ }^{1}$ Department of Public Health, School of Medicine, University of Patras, 26500 Rio Patras, Greece

${ }^{2}$ Psychiatric Hospital of Tripoli, 5th Km Tripolis-Kalamatas, 22100 Tripoli, Greece

${ }^{3}$ Health Center of Erymanthia, 25015 Erymanthia, Greece

${ }^{4}$ Department of Medical Affairs, Janssen-Cilag, 56 Eirinis Avenue, Pefki, 15121 Athens, Greece

${ }^{5}$ Department of Health Economics, National School of Public Health, 196 Alexandras Avenue, 11521 Athens, Greece

Correspondence should be addressed to Evangelia Giourou; egiourou@upatras.gr

Received 1 August 2014; Revised 20 October 2014; Accepted 22 October 2014; Published 17 November 2014

Academic Editor: Takahiro Nemoto

Copyright (c) 2014 Eleni Jelastopulu et al. This is an open access article distributed under the Creative Commons Attribution License, which permits unrestricted use, distribution, and reproduction in any medium, provided the original work is properly cited.

Introduction. Dementia's prevalence increases due to population aging. The purpose of this study was to determine the demographic profile of Greek patients with dementia and the differences in management between the urban and rural population. Methods. A cross sectional study was carried out including 161 randomly selected specialists from different regions in Greece who filled in a structured questionnaire relating to patients with dementia, regarding various sociodemographic and clinical characteristics. Results. A total of 4580 patients (52\% males) with dementia were recorded. Mean age was 73.6 years and $31 \%$ lived in rural areas. The Mini Mental Status Examination (MMSE) was used in $87 \%$ of cases. In the urban areas the diagnosis of dementia was made in an earlier stage of the disease in comparison to the rural areas $(P=0.013)$. Higher comorbidity and a higher percentage of low education were evident in rural residents $(P<0.001)$, while higher medication usage was observed in urban patients $(P=$ 0.04). Conclusions. The results implicate the need for improvement in health care delivery in Greek rural areas and health care professionals' training to achieve a proper treatment of dementias and increase the quality of life among the elderly habitants of remote areas.

\section{Introduction}

Old age seems to be the main risk factor for dementia [1, 2]. Higher prevalence rates are observed in people over 65 years old showing steadily increasing rates in each following five-year age group. About $14 \%$ of people aged 71 and older are affected by dementia, with women demonstrating higher rates in prevalence, but not in incidence [3].

Socioeconomic features [1], low educational level [3-6], cardiovascular comorbidity, and coexistence of other medical conditions increase the prevalence $[2,7]$ and the mortality of dementia [7] while the quality of life of patients decreases. Moreover, mental disorders often affect patients with dementia $[8]$ and their caregivers $[9,10]$ influencing even more their diminished quality of life and need for institutionalization. Therefore, neuropsychiatric symptoms and mental disorders of patients with dementia need to be assessed and treated independently [11].

Given the persistent prolongation of life expectancy, the impact of dementia on healthcare systems [1], on families and caregivers [12, 13], and the mortality directly linked to Alzheimer's disease (AD) and other dementias [1], it is imperative for current epidemiological data to be available to primary and secondary health care systems. A report of Turner et al. showed that general practitioners' lack of knowledge of the epidemiology of dementia had the consequence of overdiagnosing cases during their practice [14]. In a survey conducted in Greece considering the attitudes of physicians, 
caregivers, and patients regarding $\mathrm{AD}$, specialists admit lacking the expertise in distinguishing normal symptoms of aging from $\mathrm{AD}$ [15].

Epidemiological data are rare and have shown an inability to assess some basic parameters in Greece. In an epidemiological study of the municipality of Pilea, Greece, conducted on people over 70 years $(N=277)$, the estimated prevalence of Alzheimer's disease and vascular dementia was 5.3\% and $2.5 \%$, respectively [16]. In another study conducted at a primary health center in Northern Greece on people over 65 years $(N=536), 37.6 \%$ males and $41.6 \%$ females were found with cognitive impairment [17]. However, data concerning the sociodemographic and clinical profile, the therapeutic approach followed by physicians, and other important information regarding patients with dementia in Greece are scarce in the literature $[18,19]$.

In the United States one in four people older than 65 years who is living in rural environments is at greater risk of developing dementia [20]. Other studies have reported that the prevalence of cognitive impairment is higher in rural compared to urban populations [2] and there is a higher rate of hospitalization. This is probably related to distance and to limited access to health care providers compared to metropolitan citizens [21].

Moreover, most studies that include rural and remote needs within a broader national focus highlight the limited access to specialist health services and community services, compared to those living in metropolitan regions [22].

Noteworthy inefficiencies exist in the healthcare services delivery in rural areas of Greece as well. Certain disadvantages of quality and quantity of available health care providers to the residents of remote areas as well as the distinctiveness of rural-urban diversity in Greece [23] and the large proportion of elderly in rural areas, leads to the need of an update of the available epidemiological data of dementia.

The purpose of this study was to gather information to determine the demographic profile of Greek patients with dementia, to identify risk factors, and to record the screening tools used by specialists. Moreover, there was an emphasis to assess the differences in diagnostic and treatment management of dementia between urban and rural population in Greece.

\section{Methods}

2.1. Population and Setting. Medical and demographic data of 4580 patients in outpatient setting (2276 males, 2112 females, and 192 missing) who had a diagnosis of dementia by their treating physicians were included. The relevant data of participants were retrospective and they were obtained by 161 randomly selected specialists (i.e., neurologists $n=82$; psychiatrists $n=45$; neuropsychiatrists $n=32$; missing $N=$ 2 ; response rate $89.4 \%$ ) using the medical records they kept in public and private healthcare facilities all over the country. The physicians were randomly selected by the Panhellenic Medical Association's database. The treating physicians were qualified specialists (neurologists and psychiatrists) by board examinations and appropriate clinical practice. They were professionally trained in diagnosing and treating dementia according to the requirements concerning the formal residency training and the license to practice the certified specialty. The mandatory residency program requires the use of certain criteria and guidelines in diagnosing disorders. Primary care physicians were excluded because they are usually not formally trained in diagnosing and treating dementia. No other excluding criteria were used. All forms of dementia were included: Alzheimer's disease, vascular dementia, mixed, and other forms. The physicians used the information collected to fill a structured questionnaire provided by the research team.

2.2. Procedures. The study was conducted within a period of one year (1.7.2007 to 31.7.2008). During the 12-month study period, the specialist physicians enrolled a total of 4580 diagnosed patients. The patients agreed to participate in the study and provided written informed consent (mostly by their caregiver). Diagnosis of dementia was made by the treating physicians on the basis of objective cognitive tests and additional general informal tests for cognitive assessment (i.e., clinical evaluation and assessment of cognitive features such as working memory, e.g., digit span tasks, serial sevens and serial threes tasks; complex attention, e.g., months in reverse order, and orientation, e.g., time, location, and autobiographical data). For the patients' assessment, the included measure was a structured questionnaire supplied by the research team and filled by the treating doctors. The aim was to investigate the usual procedures followed by specialists in assessing and treating dementia in urban and rural areas of Greece. The questionnaire was designed by the researchers to assess differences in demographic characteristics, diagnostic procedures, and comorbid factors of dementia between urban and rural population in Greece. The questionnaire also contained items that assessed information regarding (a) sociodemographic data (age, sex, educational level, type of caregiver, and place of residence). Rural was defined as the population of those municipalities and communes in which the inhabitants of the largest population center were less than 10,000; urban was defined, respectively, as not less than 10,000 inhabitants; (b) type of dementia (Alzheimer's disease, vascular dementia, mixed form, or other); (c) comorbid factors to dementia (hypertension and other cardiological disorders, diabetes mellitus, psychiatric disorders, Parkinson disease, and other neurological disorders); (d) usage of diagnostic tools (MMSE, Clock Drawing test, Neuropsychiatry Inventory, Clinical Dementia Rating, Disability Assessment for Dementia, Global Deterioration Scale, Cambridge Cognitive Examination, or other); (e) disease's severity and symptomatology; (f) clinical and diagnostic features (i.e., clinical impression evaluation according to the physician judgment and cognitive decline evaluation according to the Mini Mental State Examination, MMSE, if the treating physician used it); and (g) drugs the treating physicians prescribed for the pharmaceutical palliative treatment of dementia, for each patient. The treating physicians were qualified specialists (i.e., neurologists and psychiatrists) professionally trained in diagnosing and treating dementia and they were randomly selected. The information collected was obtained by the 
medical records kept by the randomly selected treating physicians.

2.3. Data Analysis. All data were analyzed using descriptive statistics. Differences among subgroups (urban and rural population) were evaluated using chi-square and $t$-tests. All significance tests were two-sided using $P<0.05$ as the level of significance. Data analyses were performed using SPSS software, version 15.1.0 (SPSS, Inc., Chicago, IL, USA).

\section{Results}

3.1. Demographic and Clinical Characteristics. The majority of the studied patients $(N=4580)$ were diagnosed with Alzheimer's disease $(N=1664)$ or mixed form $(N=$ 1378). No statistical difference was observed among genders regarding dementia's forms. Patients' mean age was 74 years, while the majority of patients were over 70 years $(N=3248)$, living in urban areas $(N=2696)$, being illiterate or of lower education $(n=2020)$. Mean duration of disease was 27.6 months (median $=12$ months, maximum $=192$ months). Mean duration of treatment was 24 months. (For further details see Table 1.)

Cardiovascular risk factors, that is, hypertension $(N=$ 2209), other cardiovascular diseases $(N=1779)$, and diabetes $(N=1286)$ were the most frequent comorbidities. A positive family history of dementia was present in $34.6 \%$ of patients $(N=1587)$.

Memory decline (87.4\%) and orientation disability (61\%) were present in the vast majority of patients, while critical judgment disability (53.8\%), difficulty in planning and in complex task completion (49.8\%), and cognitive ability decline (47.4\%) followed (for further details see Table 2). Fifty-eight percent of patients were found with mood, behavior, and personality disorders while a personal history of mental disease was present in $19.5 \%$.

To assess the functional, cognitive, and behavioral status of the patients the Mini Mental Status Examination (MMSE) was the tool mostly used by physicians in their everyday practice among the screening tools (Table 3 ).

Nearly $50 \%$ of patients $(N=2172)$ received medication in the past, including nootropics $(N=963,44.3 \%)$ and acetylcholinesterase inhibitors (AchEIs) (donepezil $N=742$, $34.2 \%$; rivastigmine $N=651,30 \%)$. $71.5 \%$ were on monotherapy.

Table 4 presents the clinical impression evaluation according to the physicians' judgment and the cognitive decline evaluation according to the Mini Mental State Examination (MMSE) scale.

3.2. Rural-Urban Differences. The recruiting specialists distribution by specialty was similar between urban (51.7\% neurologists; $31.1 \%$ psychiatrists; $17.2 \%$ neuropsychiatrists) and rural areas (49.7\% neurologists; $25.3 \%$ psychiatrists; $25 \%$ neuropsychiatrists). The diagnosis of dementia in the urban areas was made in an earlier stage of the disease in comparison to the rural areas $(40.8 \%$ versus $36.2 \%, P=$ 0.013 ). Increased medication usage (three or more drugs) was
TABLE 1: Demographic and disease characteristics of study population $(n=4580)$.

\begin{tabular}{lc}
\hline Sex $(n=4388)^{*}$ & $n(\%)$ \\
\hline Male $(\%)$ & $2276(49.7)$ \\
Female $(\%)$ & $2112(46.1)$ \\
\hline Age & Years \\
\hline Mean age & 73.6 \\
\hline Nationality $(n=3758)^{*}$ & $n(\%)$ \\
\hline Greek & $3630(96.6)$ \\
Other & $128(3.4)$ \\
\hline Education ${ }^{\dagger}(n=4128)^{*}$ & $n(\%)$ \\
\hline Illiterate or low & $2020(48.9)$ \\
Middle & $1761(42.7)$ \\
Higher & $347(8.4)$ \\
\hline Residence $(n=3911)^{*}$ & $n(\%)$ \\
\hline Urban $^{*}$ & $2696(68.9)$ \\
Rural & $1215(31.1)$ \\
\hline Caregiver $(n=3805)^{*}$ & $n(\%)$ \\
\hline Relative & $3199(84.1)$ \\
Other & $606(15.9)$ \\
\hline Type of dementia $(n=4156)^{*}$ & $n(\%)$ \\
\hline Alzheimer's disease & $1664(40.0)$ \\
Vascular dementia & $1018(24.5)$ \\
Mixed form & $1378(33.2)$ \\
Other & $96(2.3)$ \\
\hline Vati &
\end{tabular}

*Valid answers.

${ }^{\dagger}$ Low education (first 6 years of formal education), middle education (secondary education consisting of 7-12 years of formal education), and higher education (tertiary education towards a degree-level qualification).

monitored in urban patients, although higher comorbidity (cardiological disorders, $P<0.001$; diabetes mellitus, $P=$ 0.001; neurological disorders, $P=0.001$ ) was evident in rural residents. Regarding the educational level, a very high percentage of low education was observed in rural population in comparison to the urban $(P<0.001)$. For further details see Table 5.

\section{Discussion}

Given the lack of epidemiological data in Greece, this study provides new insights into the epidemiological pattern of dementia in Greece. It also examines the health care inequalities as they are demonstrated through the treatment present during the time patients turned to specialists. Finally it examines the urban/rural differences in healthcare delivery in regard to patients with dementia.

In previous studies, age $[1,2]$, educational level $[3,6]$, cardiovascular comorbidity $[2,24]$, and familiar predisposition are identifiable risk factors to dementia. The present study supports these data, since the majority of patients were over 70 years, they were diagnosed with Alzheimer's disease or mixed form, they lived in urban areas and they had achieved the lower education or were illiterate. 
TABLE 2: Estimation of severity and symptomatology of dementia (\%).

\begin{tabular}{|c|c|c|c|c|c|c|}
\hline \multirow{2}{*}{ Symptomatology } & \multicolumn{6}{|c|}{ Severity ${ }^{\ddagger}$} \\
\hline & $0(\%)$ & $0.5(\%)$ & $1(\%)$ & $2(\%)$ & $3(\%)$ & Total (in \%) \\
\hline Memory decline & 0 & 1.4 & 22.1 & 59.0 & 17.4 & 87.4 \\
\hline Orientation disability & 1.6 & 3.8 & 30.0 & 49.0 & 15.5 & 61.0 \\
\hline Judgment disability & 1.2 & 4.0 & 30.3 & 48.3 & 16.1 & 53.8 \\
\hline Difficulty in planning and in complex task completion & 2.1 & 4.4 & 30.4 & 47.0 & 16.2 & 49.8 \\
\hline Cognitive ability decline & 2.6 & 4.7 & 30.9 & 46.3 & 15.5 & 47.4 \\
\hline Mood, behavioral, and personality disorders & 1.0 & 4.0 & 31.0 & 47.9 & 16.1 & 58.1 \\
\hline
\end{tabular}

${ }^{\ddagger} 0$ (no decline), 0.5 (undefined decline), 1 (minor decline), 2 (modest decline), and 3 (severe decline).

TABLE 3: Dementia screening tools used by setting (\%).

\begin{tabular}{lccc}
\hline Screening tools & Urban & Rural & Total \\
\hline Mini Mental State Examination (MMSE) & 89.9 & 92.9 & 90.9 \\
Clock Drawing test (CDT) & 41.2 & 46.3 & 42.8 \\
Neuropsychiatry Inventory (NPI) & 14.6 & 11.9 & 13.7 \\
Clinical Dementia Rating (CDR) & 2.7 & 3.0 & 2.8 \\
$\begin{array}{l}\text { Disability Assessment for Dementia } \\
\text { (DAD) }\end{array}$ & 3.6 & 1.6 & 3.0 \\
$\begin{array}{l}\text { Global Deterioration Scale, Reisberg } \\
\text { (GDS) }\end{array}$ & 2.0 & 3.3 & 2.4 \\
$\begin{array}{l}\text { Cambridge Cognitive Examination } \\
\text { (CAMCOG) }\end{array}$ & 1.1 & 1.2 & 1.2 \\
\hline
\end{tabular}

TABlE 4: Cognitive decline according to clinical impression evaluation $(\mathrm{CIE})^{\S}$ and the Mini Mental State Examination (MMSE) (in $\%)$.

\begin{tabular}{lccc}
\hline CIE & $n=2217$ & MMSE $^{\|}$ & $n=2107$ \\
\hline Very good/good & 7.8 & $\mathbf{3 0 - 2 7}$ & 2.3 \\
Satisfactory & 21.4 & $\mathbf{2 6 - 1 8}$ & 66.5 \\
Moderate & 47.0 & $\mathbf{1 7 - 1 1}$ & 26.5 \\
Bad & 23.7 & $<\mathbf{1 0}$ & 4.6 \\
\hline
\end{tabular}

${ }^{9} \mathrm{CIE}$ stands for the diagnostic procedures the treating physicians followed based on clinical features and by performing additional general additional informal tests for cognitive assessment (cognitive features were assessed such as working memory, e.g., digit span tasks, serial sevens and serial threes tasks; complex attention, e.g., months in reverse order, and orientation, e.g., time, location, and autobiographical data).

"Cut-off score under the Consensus Statements "Dementia" of the Austrian Alzheimer Society. $30-27=$ no cognitive decline; $26-18=$ mild cognitive decline; $17-11=$ moderate cognitive decline; $<10=$ severe cognitive decline.

In the literature female gender has been associated with increased risk of development of Alzheimer's disease [25, 26]. Of the total 4580 patients with all dementia forms recorded, $52 \%$ were males but no statistical difference was observed among genders regarding dementia's forms.

To assess the functional, cognitive, and behavioral status of the patients the physicians used in 90.9\% the MMSE, only in $42.8 \%$ the Clock Drawing test (CDT), and in even lower proportion other scales. MMSE and CDT are sensitive and easy to use; however, cognitive decline remains underestimated even among hospitalized elderly patients by treating physicians as a recent study of Douzenis et al.
TABlE 5: Differences of dementia patients in rural and urban areas (in \%).

\begin{tabular}{lccc}
\hline & Urban & Rural & $P$ value \\
\hline Early stage diagnosis & 40.8 & 36.2 & 0.040 \\
Lower educational level $^{* *}$ & 35.8 & 76.3 & $<0.001$ \\
Polypharmacy (3 or more medications) & 39.3 & 31.2 & 0.004 \\
Comorbidity & & & \\
$\quad$ Hypertension & 49.6 & 54.4 & 0.005 \\
Other cardiological disorders & 38.4 & 44.9 & $<0.001$ \\
Diabetes mellitus & 27.4 & 32.8 & 0.001 \\
Psychiatric disorders & 20.4 & 23.2 & 0.044 \\
Morbus Parkinson & 11.5 & 13.1 & 0.147 \\
Neurological disorders & 5.9 & 8.8 & 0.001 \\
\hline
\end{tabular}

${ }^{* *}$ First 6 years of formal education.

displayed [27]. Even if the MMSE reflects the main cognitive domains affected in $\mathrm{AD}$, additional scales are important, such as the CDT, for screening domains that are less assessed by the MMSE. Neuropsychiatric symptoms are common in dementias [28]. As this study displayed, $58.1 \%$ of patients were found with mood, behavior, and personality disorders while a personal history of mental disease was present in only $19.5 \%$. Despite that the Neuropsychiatric Inventory (NPI) provides a clinical screening examination of frequent neuropsychiatric signs which appear in dementia, it was only used in $13.5 \%$ of cases.

A large proportion $(61.2 \%)$ of the enrolled patients was newly diagnosed. According to the MMSE screening $66.5 \%$ of patients displayed Mild Cognitive Impairment (MCI; score = 26-18), which is composed of an evident cognitive decline in screening tests and others without interfering with patients' daily life. Overall prevalence of MCI among elderly is high [29]; of those a notable percentage will convert to $\mathrm{AD}$ at an accelerated rate $[1,29,30]$. Today there is a trend towards early diagnosis and treatment, towards altering progression of MCI. Patients and caregivers though abstain from confirming the existence of dementia [31] and many physicians (i.e., general practitioners and specialists) overlook symptoms of MCI attributing them to normal aging manifestations [14, 31]. While the Global Deterioration Scale (GDS) and the Clinical Dementia Rating (CDS) are important instruments in scaling patients among normal aging and severe dementia, they were only used in $2.4 \%$ and $2.8 \%$, respectively. 
Nootropics were present in medication patients received in the past, in a relatively large proportion. Furthermore, the vast majority were on monotherapy. As far as the urban/ rural diversity in health care delivery is concerned, some statistically important differences were noted. Firstly, in the rural areas dementia was diagnosed in a later stage of the disease in comparison to the urban areas, which may lead to negative effects in altering the progression of the disease itself as well as to the general well-being of patients. High mortality has been directly linked to $\mathrm{AD}$ and other dementias. Indeed, higher comorbidity was evident, in rural residents, which was expected due to certain inefficiencies of the health care system in rural areas of Greece $[23,32]$. Rural population is characterized as being less healthy overall in comparison to urban residents [33] with a higher rate of hospitalization, for a range of causes, probably related to the often limited and delayed access to health care services [21]. In a recent study of Tountas et al., rural patients' contacts with health care professionals were less than urban residents' especially among the less educated [32]. Indeed, $76.3 \%$ of patients in our study recorded in rural areas were low educated or illiterate. An increasing number of immigrants living in remote areas, carrying a low socioeconomic profile as well as the large proportion of elderly habitants of the rural areas, often illiterate [34], add to the result. Patients' and caregivers' training to recognize the early symptoms of dementia needs to be addressed in order to ensure adequate health care.

Increased medication usage (three and more drugs), as to counterbalance, was monitored in urban patients, something that enhances the need for more rational and targeted drug usage in Greece. In a recent study of Pappa et al., 2011, advanced age ( $>65$ years) visits to physician and comorbidity were associated with high rate of drug use or polypharmacy [35].

There are some limitations that merit particular consideration. The study was not a population-based cross-sectional study with a representative sample size of dementia patients. Furthermore, the physicians did not provide drop out percentages of the patients they recruited in the study. That limits the generalizability of the results. However, the specialists participating in this study were selected from various urban and rural regions from the whole country. A further weakness may arise from the fact that the questionnaire was not formally validated. On the other hand the questions were specific, well-defined, and completed by experienced and professionally qualified physicians in diagnosing and treating dementia patients. The exclusion of general practitioners may pose an extra limitation since general practitioners are more likely to encounter patients with dementia in rural areas. However, the scope of this study was to assess the demographic and clinical characteristics of patients with dementia and the diagnostic and treatment procedures used by specialists (i.e., neurologists and psychiatrists) and to describe the differences in the special care management of dementia between urban and rural population. The weak primary care sector in Greece would pose further issues concerning the validity of the diagnostic procedures followed. Furthermore comorbidities other than vascular disorders, metabolic disorders, and neuropsychiatric conditions have been correlated with dementia, such as periodontal pathogenesis or thyroid disease, which might have aggravated patients' condition. However, since there is a lack of multidisciplinary teams in the healthcare system, especially in rural areas, and also a lack of a systematic data collection, the validity of medical records could be questionable. Therefore there was an aim to describe comorbidities within broad categories of conditions which could be easily identified by the vast majority of physicians or they could be retrieved from patients' prescriptions on medications used and from medical certificates of other speciality attending physicians.

There is a growing need for interventions regarding the provision of services to patients with dementia and a growing need for the implementation of strategies to raise the awareness of the disease and to develop structures and capacities to manage the growing burden of dementia. Several issues must be addressed, such as the development of formal diagnostic and treating guidelines, the reinforcement of community support services and housing options accessible to patients and caregivers, and the creation of a national plan for dementia focused on the particular needs of the patients with dementia in Greece. This can facilitate the development of a more efficient and personalized healthcare plan for patients with dementia and high risk population living across the country.

\section{Conclusions}

In conclusion, there are considerable differences concerning dementia health care delivery among specialists practicing in urban and rural areas in Greece. Insufficiencies are observed in specialists' care to make an early diagnosis of dementia in order to prevent or slow the transition in more severe forms of the disease. There is an increasing need for improvement in health care delivery in rural areas and health care professionals' training as well as caregivers' education, in focused and proper treatment of dementias, so that substantial quality of life and general wellbeing are to be achieved even among the elderly habitants of remote areas.

\section{Ethical Approval}

Ethics approval was given from the Board of the Medical School of Patras, the Research Committee of the University of Patras, and the National Organization for Medicines, Greece. All participants and their caregivers were informed about the study and provided written informed consent for participation.

\section{Conflict of Interests}

Authors Eleftherios Moratis and Angeliki Mestousi are employed by Janssen-Cilag. All other authors declare that they have no competing interests.

\section{Authors' Contribution}

Eleni Jelastopulu participated in the design of the study, collected, analyzed, and interpreted the data, and wrote 
the first draft of the paper. Evangelia Giourou contributed to interpreting the data and to developing and writing subsequent drafts. Konstantinos Argyropoulos and Eleftheria Kariori contributed to drafting the paper and revising it critically. Eleftherios Morati and Angeliki Mestousi developed the idea for the study and were involved in the conception and design of the study and in the critical review of the paper. John Kyriopoulos participated in the design, coordinated the study, and was involved in revising the paper critically for important intellectual content. All authors read and approved the final paper. Eleni Jelastopulu is the guarantor.

\section{Acknowledgments}

This study was supported by a research grant received from the Janssen-Cilag Greece. The authors sincerely thank the physicians for all their work and contribution and all patients who kindly participated in this study.

\section{References}

[1] Alzheimer's Association, "2010 Alzheimer's disease facts and figures," Alzheimer's and Dementia, vol. 6, no. 2, pp. 158-194, 2010.

[2] B. Nunes, R. D. Silva, V. T. Cruz, J. M. Roriz, J. Pais, and M. C. Silva, "Prevalence and pattern of cognitive impairment in rural and urban populations from Northern Portugal," $B M C$ Neurology, vol. 10, article 42, 2010.

[3] B. L. Plassman, K. M. Langa, G. G. Fisher et al., "Prevalence of dementia in the United States: the aging, demographics, and memory study," Neuroepidemiology, vol. 29, no. 1-2, pp. 125-132, 2007.

[4] Y. Stern, B. Gurland, T. K. Tatemichi, M. X. Tang, D. Wilder, and R. Mayeux, "Influence of education and occupation on the incidence of Alzheimer's disease," The Journal of the American Medical Association, vol. 271, no. 13, pp. 1004-1010, 1994.

[5] D. A. Evans, L. E. Hebert, L. A. Beckett et al., "Education and other measures of socioeconomic status and risk of incident Alzheimer disease in a defined population of older persons," Archives of Neurology, vol. 54, no. 11, pp. 1399-1405, 1997.

[6] W. A. Kukull, R. Higdon, J. D. Bowen et al., "Dementia and Alzheimer disease incidence: a prospective cohort study," Archives of Neurology, vol. 59, no. 11, pp. 1737-1746, 2002.

[7] C. Helmer, P. Joly, L. Letenneur, D. Commenges, and J.-F. Dartigues, "Mortality with dementia: results from a French prospective community-based cohort," American Journal of Epidemiology, vol. 154, no. 7, pp. 642-648, 2001.

[8] J. Calleo and M. Stanley, "Anxiety disorders in later life: differentiated diagnosis and treatment strategies," Psychiatric Times, vol. 25, no. 8, pp. 24-27, 2008.

[9] K. Yaffe, P. Fox, R. Newcomer et al., "Patient and caregiver characteristics and nursing home placement in patients with dementia," The Journal of the American Medical Association, vol. 287, no. 16, pp. 2090-2097, 2002.

[10] D. H. Taylor Jr., M. Ezell, M. Kuchibhatla, T. Østbye, and E. C. Clipp, "Identifying trajectories of depressive symptoms for women caring for their husbands with dementia," Journal of the American Geriatrics Society, vol. 56, no. 2, pp. 322-327, 2008.

[11] D. Shub and M. E. Kunik, "Psychiatric comorbidity in persons with dementia: assessment and treatment strategies," Psychiatric Times, vol. 26, no. 4, pp. 32-36, 2009.
[12] M. Raivio, U. Eloniemi-Sulkava, M.-L. Laakkonen et al., "How do officially organized services meet the needs of elderly caregivers and their spouses with Alzheimer's disease?" American Journal of Alzheimer's Disease and other Dementias, vol. 22, no. 5, pp. 360-368, 2007.

[13] L. Froelich, N. Andreasen, M. Tsolaki et al., "Long-term treatment of patients with Alzheimer's disease in primary and secondary care: results from an international survey," Current Medical Research and Opinion, vol. 25, no. 12, pp. 3058-3068, 2009.

[14] S. Turner, S. Iliffe, M. Downs et al., "General practitioners' knowledge, confidence and attitudes in the diagnosis and management of dementia," Age and Ageing, vol. 33, no. 5, pp. 461-467, 2004.

[15] M. Tsolaki, S. Paraskevi, N. Degleris, and S. Karamavrou, "Attitudes and perceptions regarding alzheimer's disease in Greece," The American Journal of Alzheimer's Disease and other Dementias, vol. 24, no. 1, pp. 21-26, 2009.

[16] K. N. Fountoulakis, M. Tsolaki, R. C. Mohs, and A. Kazis, "Epidemiological Dementia Index: A screening instrument for Alzheimer's disease and other types of dementia suitable for use in populations with low education level," Dementia and Geriatric Cognitive Disorders, vol. 9, no. 6, pp. 329-338, 1998.

[17] S. Argyriadou, H. Melissopoulou, E. Krania, A. Karagiannidou, I. Vlachonicolis, and C. Lionis, "Dementia and depression: two frequent disorders of the aged in primary health care in Greece," Family Practice, vol. 18, no. 1, pp. 87-91, 2001.

[18] M. Tsolaki, K. Fountoulakis, E. Chantzi, and A. Kazis, "Risk factors for clinically diagnosed Alzheimer's disease: a case-control study of a Greek population," International Psychogeriatrics, vol. 9, no. 3, pp. 327-341, 1997.

[19] M. Tsolaki, C. Fountoulakis, I. Pavlopoulos, E. Chatzi, and A. Kazis, "Prevalence and incidence of Alzheimer's disease and other dementing disorders in Pylea, Greece," American Journal of Alzheimer's Disease, vol. 14, no. 3, pp. 138-148, 1999.

[20] R. W. Keefover, E. D. Rankin, P. M. Keyl, J. C. Wells, J. Martin, and J. Shaw, "Dementing illnesses in rural populations: the need for research and challenges confronting investigators," Journal of Rural Health, vol. 12, no. 3, pp. 178-187, 1996.

[21] J. Byles, J. Powers, C. Chojenta, and P. Warner-Smith, "Older women in Australia: ageing in urban, rural and remote environments," Australasian Journal on Ageing, vol. 25, no. 3, pp. 151-157, 2006.

[22] H. Brodaty, C. Thomson, C. Thompson, and M. Fine, "Why caregivers of people with dementia and memory loss don't use services," International Journal of Geriatric Psychiatry, vol. 20, no. 6, pp. 537-546, 2005.

[23] N. Kontodimopoulos, P. Nanos, and D. Niakas, "Balancing efficiency of health services and equity of access in remote areas in Greece," Health Policy, vol. 76, no. 1, pp. 49-57, 2006.

[24] Alzheimer's Association, "Alzheimer's disease facts and figures," Alzheimer's and Dementia, vol. 5, no. 3, pp. 234-270, 2009.

[25] N. A. Azad, M. Al Bugami, and I. Loy-English, "Gender differences in dementia risk factors," Gender Medicine, vol. 4, no. 2, pp. 120-129, 2007.

[26] P. S. Mathuranath, P. J. Cherian, R. Mathew et al., "Dementia in Kerala, South India: prevalence and influence of age, education and gender," International Journal of Geriatric Psychiatry, vol. 25, no. 3, pp. 290-297, 2010.

[27] A. Douzenis, I. Michopoulos, R. Gournellis et al., "Cognitive decline and dementia in elderly medical inpatients remain 
underestimated and underdiagnosed in a recently established university general hospital in Greece," Archives of Gerontology and Geriatrics, vol. 50, no. 2, pp. 147-150, 2010.

[28] C. G. Lyketsos, O. Lopez, B. Jones, A. L. Fitzpatrick, J. Breitner, and S. Dekosky, "Prevalence of neuropsychiatric symptoms in dementia and mild cognitive impairment: results from the cardiovascular health study," The Journal of the American Medical Association, vol. 288, no. 12, pp. 1475-1483, 2002.

[29] G. Ravaglia, P. Forti, F. Montesi et al., "Mild cognitive impairment: epidemiology and dementia risk in an elderly Italian population," Journal of the American Geriatrics Society, vol. 56, no. 1, pp. 51-58, 2008.

[30] P. Fischer, S. Jungwirth, S. Zehetmayer et al., "Conversion from subtypes of mild cognitive impairment to Alzheimer dementia," Neurology, vol. 68, no. 4, pp. 288-291, 2007.

[31] G. Waldemar, K. T. T. Phung, A. Burns et al., "Access to diagnostic evaluation and treatment for dementia in Europe," International Journal of Geriatric Psychiatry, vol. 22, no. 1, pp. 47-54, 2007.

[32] Y. Tountas, N. Oikonomou, G. Pallikarona et al., "Sociodemographic and socioeconomic determinants of health services utilization in Greece: the Hellas Health I study," Health Services Management Research, vol. 24, no. 1, pp. 8-18, 2011.

[33] M. P. R. DesMeules, C. Lagace, D. Heng, D. Manuel, and R. Pitblado, "How healthy are rural Canadians? An assessment of their health status and health determinants," in Book How Healthy Are Rural Canadians? An Assessment of Their Health Status and Health Determinants, Canadian Institute for Health Information, Ottawa, Canada, 2006.

[34] N. Bouzas, "Poverty and social exclusion in rural areas," in Book Poverty and Social Exclusion in Rural Areas, European Communities, 2008.

[35] E. Pappa, N. Kontodimopoulos, A. A. Papadopoulos, Y. Tountas, and D. Niakas, "Prescribed-drug utilization and polypharmacy in a general population in Greece: association with sociodemographic, health needs, health-services utilization, and lifestyle factors," European Journal of Clinical Pharmacology, vol. 67, no. 2, pp. 185-192, 2011. 


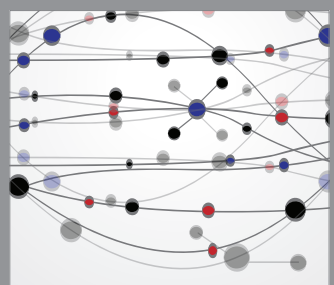

The Scientific World Journal
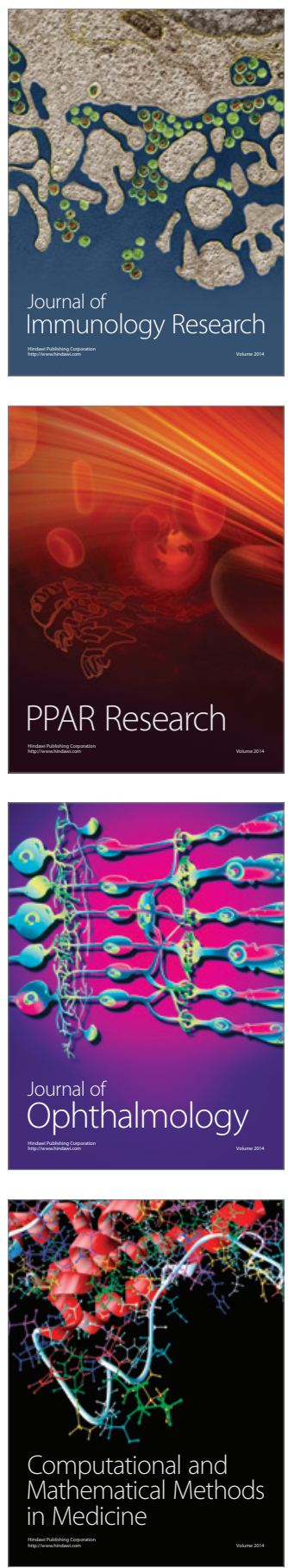

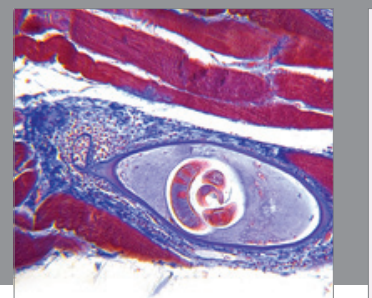

Gastroenterology

Research and Practice


\section{Hindawi}

Submit your manuscripts at

http://www.hindawi.com
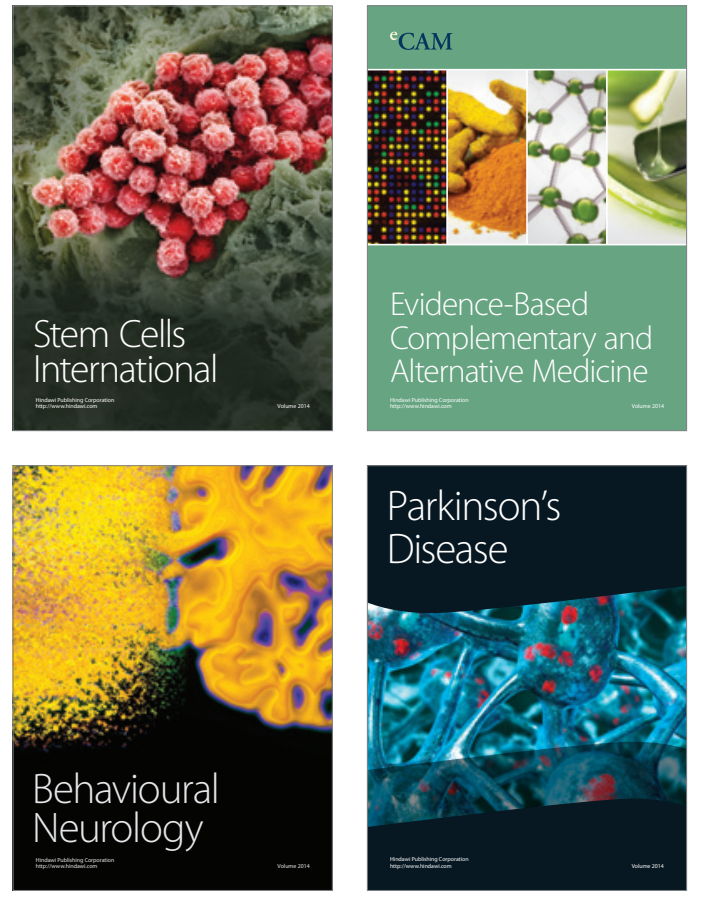
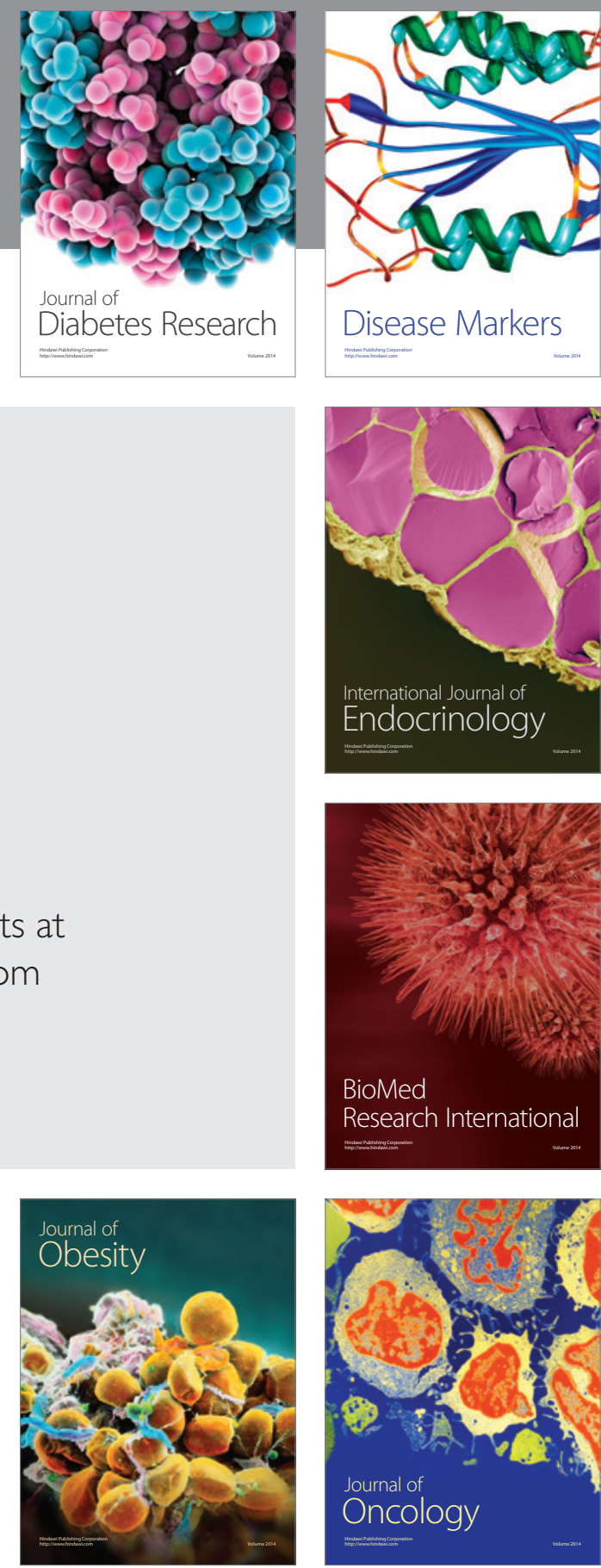

Disease Markers
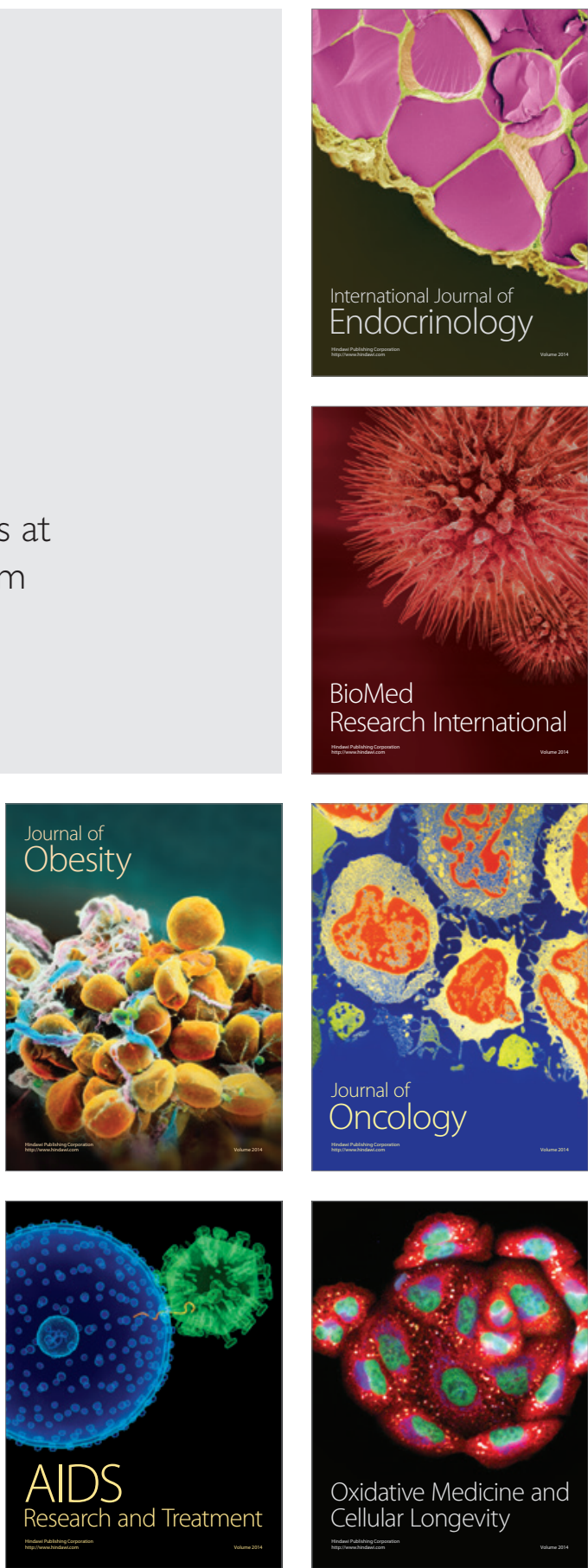